Published in final edited form as:

Nat Rev Urol. 2019 March ; 16(3): 187-200. doi:10.1038/s41585-018-0135-5.

\title{
Urologic chronic pelvic pain syndrome: insights from the MAPP Research Network
}

\author{
J. Quentin Clemens ${ }^{1,{ }^{*}}$, Chris Mullins ${ }^{2}$, A. Lenore Ackerman ${ }^{3}$, Tamara Bavendam ${ }^{2}$, Adrie van $^{2}$ \\ Bokhoven $^{4}$, Benjamin M. Ellingson ${ }^{5}$, Steven E. Harte ${ }^{6}$, Jason J. Kutch ${ }^{5,7}$, H. Henry Lai ${ }^{8}$, \\ Katherine T. Martucci ${ }^{9}$, Robert Moldwin ${ }^{10}$, Bruce D. Naliboff ${ }^{11}$, Michel Pontari ${ }^{12}$, Siobhan \\ Sutcliffe $^{13}$, J. Richard Landis ${ }^{14}$ MAPP Research Network Study Group \\ ${ }^{1}$ Department of Urology, University of Michigan, Ann Arbor, MI, USA. \\ ${ }^{2}$ National Institute of Diabetes and Digestive and Kidney Diseases, National Institutes of Health, \\ Bethesda, MD, USA. \\ ${ }^{3}$ Department of Surgery, Division of Urology, Cedars-Sinai Medical Center, Beverly Hills, CA, \\ USA.
}

${ }^{4}$ Department of Pathology, University of Colorado Anschutz Medical Campus, Aurora, CO, USA.

${ }^{5}$ Division of Digestive Diseases, University of California, Los Angeles, CA, USA.

${ }^{6}$ Departments of Anesthesiology and Medicine, University of Michigan, Ann Arbor, MI, USA.

${ }^{7}$ Division of Biokinesiology and Physical Therapy, University of Southern California, Los Angeles, CA, USA.

${ }^{8}$ Division of Urologic Surgery, Department of Surgery, and Department of Anesthesiology, Washington University School of Medicine, St Louis, MO, USA.

${ }^{9}$ Department of Anesthesiology, Perioperative and Pain Medicine, Division of Pain Medicine, Stanford University, Palo Alto, CA, USA.

${ }^{10}$ The Arthur Smith Institute for Urology, Zucker School of Medicine at Hofstra-Northwell, Lake Success, NY, USA.

${ }^{11}$ Departments of Medicine, Psychiatry and Biobehavioral Sciences, David Geffen School of Medicine at University of California, Los Angeles, CA, USA.

${ }^{12}$ Department of Urology, Lewis Katz School of Medicine at Temple University, Philadelphia, PA, USA.

\footnotetext{
*qclemens@umich.edu.

Author contributions

J.Q.C. and J.J.K. researched data for the article. All authors made substantial contributions to discussion of the article contents. J.Q.C., C.M., A.L.A., A.v.B., B.M.E., S.E.H., J.J.K., H.H.L., K.T.M., R.M., B.D.N., M.P., S.S. and J.R.L. wrote the manuscript. All authors reviewed and/or edited the manuscript before submission.

Competing interests

The authors declare no competing interests.

Publisher's note

Springer Nature remains neutral with regard to jurisdictional claims in published maps and institutional affiliations. RELATED LINKS

MAPP Research Network public website: http://www.mappnetwork.org/
} 
${ }^{13}$ Division of Public Health Sciences, Department of Surgery, and the Alvin J. Siteman Cancer Center, Washington University School of Medicine, St. Louis, MO, USA.

${ }^{14}$ Department of Biostatistics, Epidemiology and Informatics, Perelman School of Medicine at The University of Pennsylvania, Philadelphia, PA, USA.

\section{Abstract}

Urologic chronic pelvic pain syndrome (UCPPS), which encompasses interstitial cystitis/bladder pain syndrome and chronic prostatitis/chronic pelvic pain syndrome, is characterized by chronic pain in the pelvic region or genitalia that is often accompanied by urinary frequency and urgency. Despite considerable research, no definite aetiological risk factors or effective treatments have been identified. The Multidisciplinary Approach to the Study of Chronic Pelvic Pain (MAPP) Research Network uses a novel integrated strategy to characterize UCPPS as a systemic disorder that potentially involves multiple aetiologies. The first phase, MAPP I, included $>1,000$ participants who completed an intensive baseline assessment followed by a 12-month observational follow-up period. MAPP I studies showed that UCPPS pain and urinary symptoms co-vary, with only moderate correlation, and should be evaluated separately and that symptom flares are common and can differ considerably in intensity, duration and influence on quality of life. Longitudinal clinical changes in UCPPS correlated with structural and functional brain changes, and many patients experienced global multisensory hypersensitivity. Additionally, UCPPS symptom profiles were distinguishable by biological correlates, such as immune factors. These findings indicate that patients with UCPPS have objective phenotypic abnormalities and distinct biological characteristics, providing a new foundation for the study and clinical management of UCPPS.

\section{Introduction}

Urologic chronic pelvic pain syndrome (UCPPS; the Multidisciplinary Approach to the Study of Chronic Pelvic Pain (MAPP) Research Network agreed upon the definition of this disorder) encompasses two highly prevalent chronic urological pain disorders - interstitial cystitis/bladder pain syndrome (IC/BPS) in men and women and chronic prostatitis/chronic pelvic pain syndrome (CP/CPPS) in men ${ }^{1}$. IC/BPS is a debilitating bladder disorder characterized by urinary urgency, frequency and pelvic pain ${ }^{2}$. Male patients with CP/CPPS report pain in the perineum, penis, testicles or suprapubic region, which often worsens on gaculation $^{3}$. The presentation of symptoms can be quite variable among patients with UCPPS, which has led to the proposal of multiple aetiologies, some of which might be interrelated $^{1}$.

UCPPS, like many chronic pain disorders, is poorly understood, and treatment is mostly empirical, with unsatisfactory patient outcomes. Estimates of the prevalence of IC/BPS and CP/CPPS vary widely. Early studies in the 1980s suggested that IC/BPS affected as many as 500,000 US citizens ${ }^{4}$, but prevalence estimates in the past decade (using contemporary definitions of IC/BPS) now exceed 10 million (3-7\% in women and 2- 4\% in men $)^{5,6}$. Quality of life for patients with IC/BPS can be worse than that of patients with end-stage renal disease. Similar to IC/BPS, estimates of the prevalence of CP/CPPS also vary; 
community-based surveys have reported a prevalence of $8 \%{ }^{6}$, but estimates can be as high as $11.5 \%$ in men younger than age 50 years ${ }^{7}$.

The MAPP Research Network was established by the National Institute of Diabetes and Digestive and Kidney Diseases (NIDDK) of the NIH to study the aetiology and treated natural history of UCPPS ${ }^{8}$. The aim of the MAPP Research Network is to inform the treatment and management of UCPPS symptoms by improving clinical trial design and to identify clinical and biological factors and research measurements that can define clinically relevant subgroups of patients. The MAPP Network includes investigators with diverse clinical and research expertise, including urologists, gynaecologists, gastroenterologists, rheumatologists, epidemiologists, biostatisticians, molecular and cellular biologists, neurobiologists, psychologists and psychometricians, and microbiologists, among others, and it incorporates preclinical animal models of UCPPS. The group uses its diverse expertise to promote an integrated interdisciplinary characterization of UCPPS that extends beyond a sole focus on the urological system to encompass a broad spectrum of potential physiological contributing factors.

In the first phase of the MAPP Research Network (MAPP I), MAPP Network investigators launched a wide range of innovative scientific discovery efforts, moving beyond previous traditional bladder-focused and prostate-focused studies. These efforts included investigations of possible relationships between UCPPS and other nonurological chronic overlapping pain conditions (COPCs), including fibromyalgia, chronic fatigue syndrome (CFS) and irritable bowel syndrome (IBS). MAPP Network efforts included the development of an innovative cohort study; an intensive search for clinically important biomarkers and contributing molecular mechanisms; investigation of bacterial, viral and other infectious causative and/or exacerbating agents; multimodal brain imaging studies; and studies of clinically relevant animal models, among others, to better understand the underlying pathophysiology and clinical characteristics of UCPPS.

In this Review, we summarize the key insights attained to date from the major research domains of MAPP I. We discuss these findings in the context of understanding the underlying pathophysiology and diverse symptomatology of UCPPS and how they might inform clinical study design (for example, identification of new clinically relevant patient subgroups and novel targets) and improve outcomes. Finally, we highlight the potential future implications of these findings for the clinical evaluation and management of UCPPS.

\section{MAPP Network design and goals}

The current organizational structure of the MAPP Research Network includes six recruiting Discovery Sites, several specialized project-specific Discovery Sites, supporting Cores and a number of integrated subcontracts and ancillary studies, which are all based in North America ${ }^{8}$. Network Discovery Sites evaluate study participants through highly collaborative clinical and basic science studies across sites (referred to as Trans-MAPP studies). To support these efforts, the MAPP Network established two Cores. The Data Coordination Core (DCC) serves as the central site for data acquisition and storage, provides biostatistical analyses for all network-wide studies and promotes network-wide quality assurance. The 
DCC also provides administrative support, including the development and maintenance of a public website. The Tissue Analysis and Technology Core (TATC) monitors biological sample collection and provides technical expertise on a wide range of laboratory assays and/or procedures, as well as sample banking, annotation and distribution services (for a list of MAPP Research Network Discovery and Core sites and affiliated personnel, see Supplementary Box 1$)^{9}$.

During its first phase (MAPP I; 2009-2014), the MAPP Network characterized 424 participants with UCPPS (233 women and 191 men with a clinical diagnosis of IC/BPS or CP/CPPS) in a core 12-month prospective epidemiological protocol - the Trans-MAPP Epidemiology and Phenotyping (Trans-MAPP EP) Study 9,10 . The Trans-MAPP EP study design was observational (treated natural history), and the participant's physician managed clinical care, including treatment. Participants were initially assessed at baseline in a 'deep phenotyping' session that included numerous urological and nonurological patient-reported measures to determine symptom profiles, a physical examination, quantitative measures of pain sensitivity and neuroimaging via functional MRI (fMRI) to examine central nervous system (CNS) structure and function. A diverse array of biological samples (voided urine, blood and cheek swabs) was also collected for biomarker characterization and microbiome profiling. Participants with UCPPS were followed prospectively for 1 year with additional in-clinic data and sample collection and online patient-report assessments to compare baseline factors with clinical phenotype and assess changes in underlying biology over time. In addition, 415 healthy control individuals and 200 positive control participants (that is, patients with diagnosed nonurological COPCs) were characterized at baseline only, using an identical deep phenotyping session. To complement the clinical studies, investigators developed relevant animal models of UCPPS to test pathophysiological hypotheses and provide a platform for translational studies based on MAPP Network clinical insights. All studies were highly integrated to promote a systemic characterization of UCPPS and were designed to address the MAPP Network's central goals of providing new and unique insights into UCPPS, improving the evidence-based foundation for development of future clinical studies and, ultimately, improving clinical management (FIG. 1). The MAPP Network also provides a model for the integrated study of syndromic disorders, as well vast data sets and banks of biological samples for future study. In the second phase of this programme (MAPP II; 2014-2019), the MAPP Network will characterize participants with UCPPS at baseline and during an extended 36-month observational period as part of the Trans-MAPP Symptom Patterns Study (Trans-MAPP SPS) protocol. This study is ongoing and will expand greatly upon the patient phenotyping protocol from MAPP I.

\section{Clinical phenotyping}

In the MAPP Network, clinical phenotyping refers to the collection and analysis of diverse data from participants (for example, physical examination, questionnaires and medication use) to broadly describe the presentation of UCPPS for an individual or a cohort. In the first phase of studies, the MAPP Network collected extensive clinical data describing the UCPPS phenotype to correlate clinical phenotype with longitudinal symptom patterns and varied physiological and psychosocial factors and to use patient experience data to inform the development of improved research outcome measures (FIG. 2). 
A major objective of the MAPP Network is to improve the clinical evaluation of patients with UCPPS and provide supporting information to guide future studies, including clinical trials. To advance this goal, we designed studies to extensively characterize symptom profiles and reveal discrete patient subgroups (clinical phenotypes) within the broad cohort under study. In addition, this approach enabled the identification of factors associated with worsened or improved urinary pain and other clinically important symptoms over time.

To date, we have made several potentially important clinical observations among men and women with UCPPS.

\section{Nonpelvic pain and UCPPS symptom severity}

To characterize the treated natural history of UCPPS with respect to differences between localized or regional pain (pelvic pain only) and systemic pain (pelvic pain plus pain in other areas of the body) over time, a body map with a checklist of 45 body site locations was administered to each participant with UCPPS at baseline and at bimonthly assessments 9 . Approximately 25\% (108 of 424) of the participants with UCPPS reported localized abdominal-pelvic pain only at baseline, whereas the majority ( $75 \%)$ reported pain at other sites beyond the pelvis, with $\sim 33 \%$ reporting pain at $>3$ nonpelvic regions (that is, widespread pain in addition to pelvic pain) ${ }^{11}$; findings were similar in men and women. In addition, participants with widespread pain had more severe UCPPS symptoms than those with pelvic pain only. These data suggest the presence of a centralized pain phenotype in many patients with UCPPS, potentially characterized by CNS alterations or systemic pathologies. In addition, participants with pelvic pain plus widespread pain reported more psychosocial difficulties than participants with pelvic pain alone ${ }^{11}$. Whether or not other symptoms related to the centralized pain phenotype (such as somatic symptoms and sensory sensitivity) could be used to further phenotype patients is an important question for future studies.

These findings were instrumental in designing MAPP II studies, in which targeted enrolment will be performed to enrich the UCPPS cohort (50\%) with participants who report pelvic pain only at baseline. This enrichment will facilitate more detailed future comparisons with the widespread pain subgroup than was possible in MAPP I. In addition, a standardized physical examination assessing the extent and severity of pelvic floor muscle tenderness will be performed in MAPP II participants to examine whether this metric provides insight into the 'pelvic pain only' phenotype.

\section{Nonurological COPCs and UCPPS symptom severity}

Using validated symptom-based diagnostic criteria, over one-third (38\%) of the MAPP I participants with UCPPS were found to have at least one of the three common nonurological COPCs that were evaluated - IBS (22\%), fibromyalgia (4\%), CFS (3\%) or multiple COPCs $(10 \%)^{12}$. The prevalence of an additional pain syndrome was higher among women (44\%) than men (31\%), which might be due to the higher rate of these COPCs in the female population in general ${ }^{13}$. Participants with UCPPS who had additional syndromes reported more severe UCPPS symptoms and more frequent depression and anxiety than those with UCPPS alone ${ }^{12}$. These data suggest that the presence of COPCs should be considered as a 
risk factor with potential clinical significance in studies evaluating pathophysiological mechanisms and treatment of UCPPS. Further studies investigating the influence of COPCs - including the development of new COPC symptoms and their resolution - on UCPPS symptoms will be performed in MAPP II.

\section{Bladder-focused symptoms and UCPPS symptom severity}

Two RAND interstitial cystitis epidemiology (RICE) survey ${ }^{14}$ items were used to investigate bladder-focused symptoms ('bladder-focused phenotype') in the 424 MAPP I participants with UCPPS. The RICE items queried male and female participants as to whether they had 'painful bladder filling' and/or 'painful urinary urgency' ${ }^{15}$. As expected, the majority of female MAPP I participants with UCPPS ( $88 \%)$ reported painful bladder filling and/or painful urinary urgency, given that the RICE items were developed to survey women for symptoms that are consistent with IC/BPS ${ }^{14}$. Interestingly, $12 \%$ of these women did not endorse either RICE item; this UCPPS symptom group will be further characterized in MAPP II to better understand this unique and understudied phenotype. Of note, $75 \%$ of male participants with UCPPS reported painful bladder filling and/or painful urinary urgency ${ }^{15}$.

These bladder-associated symptoms are usually observed with IC/BPS; however, most of the male participants with UCPPS in MAPP I were originally diagnosed with CP/CPPS, suggesting that bladder-focused symptoms are more common in CP/CPPS than previously recognized.

These findings suggest a previously underappreciated overlap in symptomatology between CP/CPPS (men) and IC/BPS (predominantly women). Men and women with a bladderfocused phenotype characterized by painful bladder filling and/or painful urinary urgency had more severe UCPPS symptoms (pelvic pain, urinary urgency and urinary frequency), more generalized nonurological symptoms and syndromes and a poorer quality of life than those who did not endorse either of the RICE items ${ }^{15}$. Thus, bladder-focused hypersensitivity profiles are potentially important for the identification of clinically important subsets of patients with UCPPS.

These MAPP I clinical findings heavily informed the design of the MAPP II study. Important questions that will be addressed in this longer-term prospective observational cohort study include the stability of the bladder-focused phenotype and whether these phenotypes predict improvement (or worsening) of symptoms over time in the setting of usual care. In addition, patients with UCPPS in the MAPP II study will be characterized on the basis of the presence or absence of Hunner's lesions (which was not done in MAPP I) to examine the influence of these lesions on clinical phenotyping.

During a follow-up period of 12 months in MAPP I, $60 \%$ of participants with UCPPS had stable symptoms, whereas $20 \%$ demonstrated UCPPS symptom improvement and $20 \%$ exhibited symptom deterioration (worsening) ${ }^{16}$. The extent of widespread pain, number of nonurological symptoms and poor overall health status were predictive of poor outcomes. Importantly, these observations were made while participants were receiving usual care for their symptoms, and medications and/or treatments were recorded only every 2 months. Thus, it was not possible to determine the impact of individual treatments on symptom trajectories. In MAPP II studies, participants with UCPPS will be followed for a longer 
period of time (36 months) than in MAPP I, and UCPPS treatments will be prospectively recorded to determine whether specific phenotypic characteristics correlate with treatment response to specific agents. We hope that the findings from these studies will lead to focused clinical trials in which specific agents can be targeted to specific patient phenotypes.

\section{Assessment of urological pain and urinary symptoms}

Using questionnaire responses from participants with UCPPS, principal component and exploratory factor analyses were performed to determine a simple yet comprehensive set of symptom indices to characterize and quantify UCPPS symptoms. Two factors - pelvic pain severity and urinary symptom severity - provided the best psychometric description of items from the multiple symptom questionnaires that were administered ${ }^{17}$. Latent factors were used to create two separate symptom indices, one for UCPPS pain and another for urinary symptoms. UCPPS pain, but not urinary symptoms, was associated with symptoms of depression, suggesting that urological pain and urinary symptoms vary in their influence on a patient's quality of life. On the basis of these findings, UCPPS pain and urinary symptoms should be measured separately using the Genitourinary Pain Index (GUPI) ${ }^{16}$ and Interstitial Cystitis Symptom Index (ICSI) ${ }^{18}$ symptom subscales, which would provide a brief but discerning UCPPS symptom severity assessment based on well-accepted instruments. Future MAPP Network studies will investigate how these symptom indices change over time, their relationship to other important physiological factors (for example, catastrophizing and anxiety) and whether they can predict disease outcomes.

Collectively, these insights suggest that at least three critical factors should be prioritized in the clinical phenotyping of patients with UCPPS - pain localization (pelvic pain only versus pelvic pain plus widespread pain), the presence or absence of the full range of COPCs and bladder-focused symptoms (TABLE 1). Another major implication of this work is that clinicians should consider abandoning the use of comprehensive symptom indices that report total scores for UCPPS and instead evaluate urological pain and urinary symptoms separately.

\section{UCPPS symptom flares}

Patients with UCPPS frequently report 'symptom flares' (acute worsening of symptoms) ${ }^{19}$. However, no previous studies have systematically examined the characteristics of UCPPS symptom flares or the influence that flares have on the patient experience. To develop an improved, patient-centric understanding of symptom flares, the MAPP Network conducted a multisite focus group study with patients with $\mathrm{UCPPS}^{20}$ and two smaller site-specific studies $^{21,22}$. These studies revealed the presence of multiple types of UCPPS symptom flares, which vary in symptom presentation, severity and duration (from minutes to days or longer). Furthermore, increased flare frequency was an important component of overall symptom severity and morbidity. Focus group participants also indicated that flare predictability is a major concern for patients; flares that arise without any warning or explanation lead to increased distress and have a negative influence on a patients' daily function. A resultant and clinically significant effect of frequent flares is social avoidance and social isolation ${ }^{20}$. 
Another important focus of the MAPP Network studies is to explore flare triggers. Although several previous studies ${ }^{23-26}$ have asked patients what they believe triggers their flares, few studies have examined these possible triggers empirically. Thus, as part of the Trans-MAPP EP Study, we performed a case-crossover study ${ }^{27}$ that compared participants' experiences before their first three flares with their experiences before three random biweekly visits during which they reported not having flares; this design enabled each participant to serve as his or her own control. At each of the case-crossover study assessments, participants completed a 'flare risk factor' questionnaire that assessed factors that could potentially trigger flares (for example, foods and drinks, physical activity, sexual activity and infections). Of the factors examined, recent sexual activity was the only factor associated with flare onset in the full study population ${ }^{28}$. However, in subpopulations that were thought to be highly susceptible to certain triggers, additional associations were observed for several foods and drinks, as well as for abdominal exercises. For example, in the subset of participants who identified dietary factors as a cause for UCPPS symptom flares, consumption of tomatoes, yogurt, spicy foods, alcohol, caffeinated beverages and carbonated beverages was associated with flare onset. These findings suggest that flare triggers are specific to certain patients and, therefore, that adoption of global flare prevention strategies would be too restrictive for many patients.

Finally, data from the case-crossover study also enabled assessment of the incidence of UCPPS symptom flares over the entire study period. Almost $80 \%$ of all participants with UCPPS reported at least one symptom flare during the 12-month study period ${ }^{27}$. Specifically, 24\% reported no flares during the follow-up period, $23 \%$ reported one flare, $28 \%$ reported two to three flares, and $24 \%$ reported four or more flares (J.Q.C., H.H.L., B.D.N., S.S. and J.R.L., unpublished observations). Flares were more frequently reported in women (mean 2.8 flares) than in men (mean 1.9 flares) (J.Q.C., H.H.L., B.D.N., S.S. and J.R.L., unpublished observations). Furthermore, flares were more common in participants who endorsed body map pain sites beyond the pelvic region than those in the subgroup with pelvic pain only and in participants with the most severe bladder symptoms (J.Q.C., H.H.L., B.D.N., S.S. and J.R.L., unpublished observations).

Questions related to flares and their influence, including the use of mobile technology applications and ecological momentary assessment to examine short-term (hourly) flare patterns and their associated risk factors, will be further addressed in ongoing MAPP Network studies.

\section{Psychosocial factors}

Psychosocial factors have a major influence on quality of life and other outcomes related to chronic pain ${ }^{29-31}$. The MAPP Network study is the first to directly assess a large cohort of male and female patients with UCPPS using a broad range of psychosocial variables (FIG. 2). Participants with UCPPS reported more psychosocial difficulties, including higher levels of current and lifetime stress, poorer coping and more self-reported cognitive deficits, than healthy control individuals matched for age and sex who did not have pain ${ }^{32}$. In addition, both sexes showed similar levels of psychosocial problems, although women had higher levels of childhood adversity and widespread discomfort than men ${ }^{32}$. Interestingly, the level 
of psychosocial problems was not solely attributed to the severity of UCPPS symptoms, suggesting that a reduction in the symptoms associated with UCPPS alone would be insufficient to produce clinically significant improvements in quality of life for some patients. Perhaps unsurprisingly, the presence of nonurological COPCs (fibromyalgia, IBS and/or CFS) in the UCPPS cohort was associated with higher rates of depression and anxiety, as well as greater UCPPS symptom severity ${ }^{12}$. Finally, several psychological variables - such as catastrophizing, a lowered sense of mental and, particularly, physical well-being, and the presence of life stressors - were associated with a low probability of symptom improvement over a 12-month period ${ }^{33}$. MAPP II studies will help to determine the stability of these patterns by assessing psychosocial variables in participants with UCPPS over a 36-month period.

\section{Neuroimaging}

Before the MAPP Network, few published studies had assessed alterations in brain structure or function in patients with UCPPS. These early studies primarily aimed to demonstrate the feasibility of neuroimaging to study chronic urological pain disorders ${ }^{34,} 35$ and were limited by their small sample sizes, restriction to male patients only and lack of convergent findings of altered brain structure and function. Concurrently, research in other chronic pain conditions had started to demonstrate that brain structure and function are important factors to be considered in persistent pain, as specific brain phenotypes can perhaps promote or inhibit recovery from pain ${ }^{36-39}$. The MAPP Network, when established, recognized the crucial importance of assessing the involvement of brain-level changes as part of the central Trans-MAPP EP Study to enable a comprehensive characterization of UCPPS beyond the bladder or prostate 8,40 .

To substantially increase the sample size for studies assessing the contributions of brain structure and/or function to UCPPS, the MAPP Network developed an innovative protocol that standardized fMRI data acquisition across sites ${ }^{41}$. This approach has yielded a common neuroimaging data set integrated with clinical data and other phenotyping domains that is unprecedented in the field. Importantly, the MAPP Network treated sex as a crucial biological variable by focusing neuroimaging data analyses exclusively on either women $^{42-46}$ or men ${ }^{47}$ or by stratifying participants by sex ${ }^{48-50}$. The MAPP Network neuroimaging study was also the first to use multiple neuroimaging modalities in a common protocol for assessing brain correlates of UCPPS urological and pain symptoms (FIG. 2). These MRI modalities included T1-weighted imaging for grey matter structure, diffusion tensor imaging (DTI) for white matter structure and resting-state fMRI (rs-fMRI) for grey matter function. This approach enables the potential identification of independent sources of complementary evidence that point to brain networks with altered structure and function in UCPPS.

Convergent findings of altered brain structure and function (in the context of a comparison between participants with UCPPS and controls) were reported in MAPP I neuroimaging studies (FIG. 3). Differences between participants with UCPPS and healthy control individuals were particularly apparent in medial areas of the motor and sensory cortex, the right posterior insula and the periaqueductal grey (PAG) area of the brainstem ${ }^{51,52}$. These 
motor and sensory areas are consistent with the sensorimotor representation of the pelvic area. The right posterior insula consistently activates during intravesical infusion of fluid ${ }^{53,54}$ and is thought to be a primary viscerosensory region associated with the bladder ${ }^{54}$. The PAG has a crucial role in both the control of urination ${ }^{55}$ and the descending modulation of nociceptive signals ${ }^{56}$. Findings of altered grey matter structure, white matter structure and resting-state function are particularly convergent in the supplementary motor area (SMA), a region that has a key role in providing corticospinal input to active pelvic floor muscles ${ }^{54,55,57,58}$. These findings collectively enable us to make the preliminary conclusion that UCPPS involves disturbances in brain-level sensorimotor systems regulating urine storage and that these disturbances are powerful enough to produce differences not only in brain function but also in brain structure. Thus, it seems that dysfunction associated with UCPPS is not confined to either the end-organ (bladder or prostate) or spinal-level neural systems in many patients but involves measurable brain-level changes. MAPP II studies will determine whether these findings can be reproduced in a separate cohort of participants with UCPPS.

Comparison of participants with UCPPS with healthy control individuals provides an important first-level understanding of the neural contributions to the syndrome. However, MAPP Network neuroimaging data also provide a novel means of identifying underlying neurobiological factors for patients with UCPPS who display different clinical features. One such clinical consideration is the longitudinal progression of symptoms; for some participants with UCPPS, symptoms were relatively constant, whereas symptoms improved and/or worsened over time for others ${ }^{33}$. In 2017, the MAPP Network published the first study showing that rs-fMRI data are predictive of trends in UCPPS pain symptom severity in the 3 months following the scan ${ }^{50}$. Importantly, the neuroimaging readings were conducted in a blinded manner in these studies. Participants with UCPPS whose pain symptoms improved had stronger functional connectivity in the left frontoparietal brain network than those whose pain symptoms remained constant or worsened ${ }^{50}$. These differences in brain function might reflect neural systems that allow the regulation of a patient's attention to their symptoms. Furthermore, neuroimaging findings from other MAPP Network studies have revealed that UCPPS involves structural, chemical and functional brain changes consistent with other chronic pain conditions, such as fibromyalgia ${ }^{43,44,46,59}$. Network studies addressing the possible contributions of a centralized pain phenotype in UCPPS have also shown changes in functional connectivity patterns associated with the presence of widespread pain versus pain localized to the pelvis, providing a neurobiological correlate to a potential subgroup of patients with UCPPS ${ }^{50,60}$. Ongoing studies, including longitudinal neuroimaging and a natural bladder-filling paradigm (via diuresis), are underway in MAPP II to extend these observations ${ }^{61}$.

\section{Quantitative sensory testing}

Another characteristic of patients with centralized chronic pain is a generalized or global abnormality of sensory processing ${ }^{62,63}$. In the MAPP Network, sensory processing abnormality was assessed using quantitative sensory testing (QST) (FIG. 2), which refers to experimental procedures that assess evoked perceptual responses to quantifiable physical stimuli delivered to the body (for example, blunt thumbnail pressure combined with 
graphical touch screen interface to measure pain level). Data from QST studies suggest a normal distribution of pain sensitivity across the general population, with the majority of individuals with centralized pain exhibiting marked hypersensitivity (falling on the right side of the normal distribution curve $)^{64-66}$. Importantly, hypersensitivity can be present in both symptomatic and nonsymptomatic body sites, the latter of which is strongly suggestive of CNS-related pathophysiological mechanisms and occurs in many chronic pain conditions, such as fibromyalgia ${ }^{67,68}$, IBS $^{69}$, temporomandibular joint (TMJ) disorder ${ }^{70,71}$ and lowerback pain ${ }^{72}$.

Previous research suggests that some patients with UCPPS also exhibit QST findings that are consistent with a centralized pain phenotype, including generalized pain hypersensitivity and deficient endogenous pain inhibition ${ }^{73-77}$. However, these studies mostly had small sample sizes and were limited to women, making it difficult to explore potential sex and subgroup differences in UCPPS sensory processing. MAPP I included the largest examination of generalized pain sensitivity in UCPPS to date. In MAPP I, an automated QST system ${ }^{78}$ delivered computer-controlled pressure stimuli to the thumbnail as a validated model of centralized pain ${ }^{63,79-81}$. Analyses revealed a significant $(\mathrm{P}<0.05)$ increase in pressure pain sensitivity in participants with UCPPS ( $n=153 ; 51 \%$ female) compared with healthy control individuals ( $n=100 ; 59 \%$ female) (S.E.H., H.H.L., S.S., B.D.N. and J.R.L., unpublished observations). However, participants with UCPPS were generally less sensitive to pressure pain than a mixed pain comparison group that included participants with fibromyalgia, CFS and IBS ( $n=35 ; 77 \%$ female). Additional network studies examining auditory sensitivity and endogenous analgesia in UCPPS were also conducted. Participants with UCPPS showed increased sensitivity to unpleasant auditory stimuli compared with healthy control individuals, and an association between deficient endogenous pain inhibition and self-reported nonurological pain was observed (S.E.H., H.H.L., S.S., B.D.N. and J.R.L., unpublished observations). These data indicate the presence of a global multisensory hypersensitivity for unpleasant or painful stimuli in UCPPS, providing further evidence for a centralized pain phenotype. QST findings were also examined as a predictor of longitudinal symptom change in participants with UCPPS. Exploratory analyses indicated that low pressure pain sensitivity at baseline was associated with a high probability of improvement in urogenital pain severity over a 12-month period (S.E.H., H.H.L., S.S., B.D.N. and J.R.L., unpublished observations).

A broadened arsenal of QST methods - including analyses of endogenous analgesia and temporal summation of pain - is being used in MAPP II to enable a more comprehensive evaluation of sensory processing in UCPPS than was attainable in MAPP I. In addition, a considerably larger group of participants with UCPPS will undergo assessments with both QST and neuroimaging in MAPP II. Together, these data will provide greater insight into the neurobiological mechanisms underpinning UCPPS sensory symptoms and the prediction of longitudinal symptom patterns.

\section{Molecular phenotyping}

Within the MAPP Network, molecular phenotyping refers to the investigation of molecular and pathobiological correlates of UCPPS. Several approaches have been used to study 
biomarkers in the MAPP Network using a variety of collected biological samples (FIG. 2). MAPP Network studies were initiated with a comprehensive literature review that identified a panel of candidate biomarkers for UCPPS, including matrix metalloproteinase 2 (MMP2), MMP9, neutrophil gelatinase-associated lipocalin (NGAL), the MMP9-NGAL complex, vascular endothelial growth factor (VEGF) and VEGF receptor 1 (VEGFR1) ${ }^{82}$. These factors were measured in the urine of participants with UCPPS, positive control individuals (that is, participants with one or more COPC) and healthy control individuals in the TransMAPP EP Study. Normalized urine concentrations of VEGF $(P<0.0001)$, VEGFR1 ( $P=$ $0.004)$ and MMP9 $(P=0.001)$ in men and $\operatorname{VEGF}(P=0.0076)$ in women were significantly higher in participants with UCPPS than in healthy control individuals ${ }^{82}$. Differences in the urine levels of MMP9, MMP9-NGAL and VEGFR1 in men and all assayed biomarkers in women were associated with the severity of clinical symptoms. These findings might have importance in understanding the varied bladder pain phenotypes (pain with bladder filling or pain relief by bladder emptying observed in UCPPS), as well as potential sex differences in the underlying pathophysiological mechanisms. One proposed mechanism in UCPPS is alteration of bladder blood flow ${ }^{83}$, given that VEGF is involved in the development and regulation of blood vessels. VEGF and MMP9 have a role in neuropathic pain ${ }^{84,85}$, suggesting a possible neuropathic aetiology for UCPPS in some participants. MMP9-NGAL is considered a marker of infection or inflammation; thus, the observed elevations in UCPPS also suggest an inflammatory aetiology ${ }^{86}$. To discover novel urinary biomarkers of UCPPS, a comparative quantitative mass spectrometry (MS)-based analysis is underway and has identified a number of new candidates that are differentially expressed in the urine of patients with UCPPS and healthy control individuals.

MAPP Network studies have also found that female participants with UCPPS exhibit an exaggerated inflammatory response compared with healthy control individuals, as evidenced by a greater inflammatory response to stimulation of Toll-like receptor 2 (TLR2) and TLR4 in patient-derived peripheral blood mononuclear cells (PBMCs) ${ }^{87}$. TLR2 and TLR4, which recognize specific molecular components of microorganisms (such as bacteria) to activate the innate immune system ${ }^{88}$, have an important non-canonical role in pain sensitization at the level of the spinal cord ${ }^{89}$. Response to TLR4 stimulation also positively correlated with urological pain severity and distinguished participants with UCPPS who had comorbid COPCs and widespread pain from those with pain confined to the pelvic region and/or bladder ${ }^{90}$. The TLR4 response was also associated with low pressure pain thresholds measured at the thumbnail via $\mathrm{QST}^{90}$. These findings represent novel insights into pathophysiological mechanisms underpinning UCPPS, given that very few biomarkers have been reported to correlate with pain in patients with UCPPS and that previous studies have not used such a stimulated biological response method to examine immune system correlates. In addition, these analyses also revealed abnormalities in the hypothalamicpituitary-adrenal (HPA) axis in participants with UCPPS and showed that a combination of HPA dysregulation and inflammatory responsiveness was a strong predictor of pelvic pain ${ }^{87}$. This finding is similar to that reported in other systemic chronic pain conditions ${ }^{91}$ and lends further support to the hypothesis that UCPPS has systemic contributors beyond urological organs or tissues in some patients. Furthermore, increased TLR2 and TLR4 responsiveness was associated with decreased improvement in pelvic pain over time, whereas TLR4 
responsiveness alone was associated only with decreased improvement in urinary symptoms ${ }^{92}$. This association between response and specific phenotypes will be studied further in MAPP II.

The MAPP Network also conducted metabolomics studies of urine specimens using MSbased global metabolite profiling approaches - multivariate analysis of liquid chromatography (LC)-MS and LC-MS with tandem MS (LC-MS/MS) data - to discern small-molecule correlates of UCPPS (compared with controls) and symptom profiles ${ }^{93}$. These studies found that the testosterone metabolite etiocholan-3a-ol-17-one sulfate (EtioS) distinguished female participants with UCPPS who have a high symptom burden from those with a low symptom burden at follow-up periods of 6 and 12 months ${ }^{94}$. In previous studies, Etio-S has been associated with inflammatory responses following administration in humans ${ }^{94}$. Further investigation of this novel finding might lead to previously unconsidered mechanisms that contribute to UCPPS symptoms.

\section{Contributions of the microbiome}

The expansion of pathogenic bacteria in the bladder and urinary tract is well known to lead to common urological disorders, including cystitis, pyelonephritis and prostatitis ${ }^{95,96}$. However, past investigations in IC/BPS and CP/CPPS, including published studies of antibiotic use as a medical intervention, have been inconclusive and even contradictory in demonstrating the contribution of microbial infection to symptoms ${ }^{97-100}$. Over the past decade, paradigm-shifting analyses using culture-independent next-generation sequencing (NGS) and modified, sensitive culture techniques have identified a putative bladder microbiome ${ }^{101,102}$ and have subsequently linked decreases in bacterial diversity in the bladder to multiple urological conditions, including IC/BPS ${ }^{103,104}$. These insights and the evolution of new and increasingly powerful high-throughput methodologies have renewed interest in addressing long-standing questions of the role of the microbiome in urological disorders, including UCPPS.

To overcome previous methodological limitations in studies of UCPPS, the MAPP Network used a novel state-of-the-art, culture-independent molecular method (Ibis T-5000 Universal Biosensor technology) to identify associations between microbial profiles using a series of urine samples (FIG. 2 ) and clinical phenotyping data ${ }^{105}$. Significant differences in the overall microbial genus $(P=0.004)$ and species $(P=0.002)$ composition were observed between first-void urine samples from male participants with UCPPS and healthy control individuals, with Burkholderi a cenocepacia noted to be top differentially abundant species in UCPPS ${ }^{105}$. In addition, Lactobacillus gasseri was found to be over-represented in both first-void (initial $10 \mathrm{ml}$ of urine) and midstream urine samples from female participants with UCPPS compared with healthy control individuals. These findings constitute the first reports of an association between specific bacterial species and UCPPS, supporting a potential pathophysiological role for resident urinary tract microorganisms in this condition.

In addition, women who reported UCPPS symptom flares at baseline were more likely to have yeast (including Candida spp. and Saccharomyces spp.) present in midstream urine specimens than women who did not report a flare ${ }^{106}$, prompting the need for further 
investigation of the role of fungi in urological diseases. Using an independent, NGS-based approach optimized for the detection of urinary fungi, the MAPP Network identified and characterized a remarkable community of fungi in the urinary tract that has greater diversity than the neighbouring sites of the vagina and inguinal crease $\operatorname{skin}^{107}$. A profound decrease in this fungal diversity was also observed in patients with UCPPS, and increases in the abundance of fungi of order Saccharomycetales correlated with increased pain severity. These intriguing fungal findings will be expanded upon in MAPP II, which will analyse both bacterial and fungal differences that occur in participants with UCPPS at multiple time points over an extended observation period of 36 months.

\section{Animal model studies}

To provide an opportunity for bidirectional testing of mechanistic and clinical insights, a multidisciplinary team of MAPP Network investigators defined key phenotypic criteria for establishing rodent models of UCPPS ${ }^{108}$. Patient symptoms were initially prioritized on the basis of their clinical significance, with pelvic pain and urinary frequency selected as hallmark human symptoms for use in evaluating proposed animal models (FIG. 2). Methods for measuring rodent correlates of patient symptoms were then applied across a number of transgenic or induced rodent models of UCPPS with the goal of identifying animals that exhibited these key symptom correlates, therefore improving the translational validity of bidirectional experimental findings. In these studies ${ }^{108-116}$, bladder nociception was quantified using visceromotor response to bladder distention, referred mechanical allodynia (also known as tactile allodynia) of the pelvis was assessed by applying von Frey filaments to the lower abdomen, and urinary frequency was assessed both as free voiding using micturition cages and voiding spot assay and in response to filling cystometry.

On the basis of these validation criteria, cyclophosphamide-induced (chemical cystitis), UTI (acute infection and post-UTI pain), chronic stress-water avoidance (stress-induced pain), transgenic URO-OVA mouse (autoimmune cystitis in the bladder; induced in animals expressing self ovalbumin under a bladder urothelial cell-specific promoter by introduction of activated OVA-specific T cells), transgenic URO-MCP1 mouse (monocyte chemoattractant protein 1 (MCP1; also known as CCL2) expression in the urothelium producing bladder hypersensitivity) and pseudorabies virus (PRV; neurogenic cystitis) models were selected ${ }^{108}$. Importantly, these models are founded on leading hypotheses of underlying pathophysiological mechanisms in human UCPPS. All models examined demonstrated pelvic nociception (referred mechanical allodynia) with von Frey filament testing ${ }^{117}$. Nociception to bladder distention, pelvic nociception and urinary frequency were recapitulated in the cyclophosphamide and chronic stress-water avoidance models ${ }^{109}$, pelvic nociception and urinary frequency were present in the URO-OVA ${ }^{118}$ and URO-MCP1 models ${ }^{110}$, and the UTI and PRV models demonstrated bladder hyperalgesia ${ }^{111,112}$.

Following validation, additional mechanistic studies using these UCPPS models were performed. Results from these studies highlighted the importance of central sensitization, inflammation-associated bladder dysfunction and pelvic visceral organ cross-sensitization in the pathogenesis of pelvic pain. For example, MAPP Network investigators showed that chronic pelvic allodynia could develop after the resolution of UTI caused by a strain of 
Escherichia coli lacking O-antigen expression (post-UTI chronic pain) ${ }^{113}$. Moreover, treatment of the bladder with protamine sulfate was found to be analgesic and tissue protective and suppressed the expression of inflammatory cytokines that are normally associated with nociception after an E. coli $\mathrm{UTI}^{114}$. The importance of metabotropic glutamate receptor 5 (mGluR5)-extracellular signalling regulated kinase (ERK) signalling pathway activation at the levels of the spinal cord and the central nucleus of the amygdala in the development of central sensitization was also revealed ${ }^{109,115,116}$.

In MAPP II, investigators are building on the observations of the first-phase studies to enable further analysis of new clinical insights in validated UCPPS models. Areas of special interest will include assessing the in vivo contribution of biological factors (supported by evidence from biomarker studies) to nociception and urinary dysfunction; rodent neuroimaging studies to examine the effects of stress on functional brain reorganization, nociception and bladder function; and comparison with insights from the human neuroimaging studies.

\section{Implications of MAPP Network findings}

A major objective of MAPP Network studies is to improve the clinical evaluation of patients with UCPPS and to provide supporting information to guide future studies, including clinical trials. To advance this goal, we designed studies to elaborate on symptom profiles and reveal discrete patient subgroups (clinical phenotypes) within the broad cohort under study. In addition, this approach allows identification of factors associated with UCPPS symptom changes over time. To date, we have made a number of potentially important clinical observations among men and women with UCPPS, which are summarized below.

\section{Future clinical trial design}

MAPP Network findings indicate that specific clinical factors that are easily measurable including the presence of pain beyond the pelvis, the presence of COPCs and the severity of bladder-focused symptoms (particularly pain with bladder filling) ${ }^{15}$ - have a clear influence on symptom trajectories over time. These findings suggest that future clinical trials should consider stratifying patients according to these factors, as they might influence treatment efficacy. Furthermore, the correlations between widespread pain and neuroimaging and/or QST findings ${ }^{50,60}$ suggest that a subset of patients with UCPPS have a centralized pain phenotype and that they might respond to centrally acting treatments. This possible relationship between phenotypic characteristics (central versus peripheral pain mechanisms) and response to treatment type (centrally acting versus peripherally acting therapies) is a major focus of MAPP II studies. In addition, MAPP Network studies have revealed the importance of measuring urological pain and urinary symptoms separately, rather than with a composite score ${ }^{17}$. Specific therapies could possibly lead to improved outcomes for patients with one type of symptom but not necessarily both; such relationships might have been missed in previous clinical trials in which the primary outcome measure was assessed using composite scores. 


\section{Multimodal management}

MAPP Network studies have provided novel insights into symptom severity in UCPPS. Indeed, patients with nonurological COPCs (such as IBS, CFS and fibromyalgia) or those who present with widespread pain in addition to pelvic pain frequently endorse the highest measure of UCPPS pain severity in administered questionnaires and, perhaps expectedly, have a higher risk of depression and anxiety and a poorer quality of life than those with UCPPS alone and pelvic pain alone, respectively ${ }^{12}$. The high percentage of participants with UCPPS who had accompanying nonurological COPCs and psychosocial comorbidities ${ }^{12 \cdot 32}$ suggests an important role for multimodal management and the frequent need for therapies that extend beyond the pelvic viscera. This information is critically important for clinicians from the perspective of identifying other therapeutic targets and patient counselling. This finding also raises the question as to whether a patient's symptoms would be best managed in medical environments that can address these varied needs using holistic approaches that take into account psychosocial comorbidities, COPCs and patient experiences with symptom flares. Interventions that aim to improve a patients' ability to cope with and self-manage symptoms and their effects, such as cognitive behavioural therapy, have shown promise in other chronic pain conditions and deserve further study in UCPPS ${ }^{119}$. The attainment of highly reliable information about the prognosis of UCPPS symptoms over time (for example, the likelihood of worsening or improving) is one of the major clinical goals of the MAPP Network and is being further addressed in the extended observational period of the MAPP II study.

\section{Aetiological crossover}

In standard urological practice, men tend to present with pelvic pain of unknown aetiology and are, therefore, classified with the general diagnosis of 'prostatitis' (Prostatitis Type III; $\mathrm{CP} / \mathrm{CPPS}$ ), a condition that has a limited number of effective therapies ${ }^{3}$. Data generated within the MAPP Network have revealed a strikingly high prevalence of painful bladder filling and/or painful urinary urgency in male participants with UCPPS ${ }^{15}$, suggesting the existence of a clinical and perhaps aetiological crossover between IC/BPS (predominantly women) and CP/CPPS (men). This finding illustrates the importance for clinicians of querying male patients with chronic pelvic pain about bladder symptoms, as an affirmative response might lead to a change in diagnosis and subsequent therapy assignment.

\section{Bladder-focused pain and urinary urgency frequency outcomes}

To date, the combination of pelvic pain, urinary symptoms and other measurements (such as quality of life) to derive a composite score has been a common practice; these composite scores are used to assess outcome measures in clinical trials and to monitor clinical progress ${ }^{120,121}$. One of the most important findings of MAPP I is that this approach might not be appropriate. The MAPP Network has shown that UCPPS pain and urinary symptoms (urgency and/or frequency) have a different quality of life influence and severity pattern over time $^{33}$ and therefore should be assessed separately in patients with UCPPS in research and, potentially, clinical settings. This finding has major implications for the design of research studies and patient follow-up procedures in standard practice. In addition, new instruments 
developed on the basis of these findings might influence how outcomes (symptom improvement) are assessed and how patient cohorts are screened in future clinical trials.

\section{Symptom flares}

Once a diagnosis of UCPPS has been made, clinicians typically begin therapy to treat pain that is known to be chronic but is often assumed to be stable in its severity and characteristics ${ }^{122}$. MAPP Network symptom flare data have dispelled this traditional notion by demonstrating that flares occurred in almost all participants with widely different frequencies, severities, durations, symptoms, influences on quality of life and, possibly, triggers ${ }^{20,22}$. The finding that symptom flares are common and can have a very profound psychosocial influence strongly suggests that their assessment needs to be a priority in clinical evaluation and management. An understanding of flare characteristics can be helpful for the selection of appropriate therapies that aim to mitigate flare-specific symptoms. For example, factors that trigger symptom flares (for example, foods, beverages and physical activity) might need to be modified in a patient-centric manner and as part of a personalized approach to management. In addition, individualized strategies for addressing flares (a 'flare plan') need to be developed on the basis of an understanding of the patient experience rather than preconceived management approaches. These approaches might range from simple strategies, such as taking a warm bath, controlling constipation with diet or medications or carrying medications at all times, to more aggressive interventions, such as intravesical lidocaine instillations.

\section{Psychosocial factors}

One of the primary clinical goals for patients with UCPPS is to improve their quality of life. Many clinicians (and patients) assume that once symptoms improve, quality of life will similarly improve. MAPP Network findings have demonstrated that this assumption might not be the case if the patient has substantial coexisting psychosocial comorbidities or severe nonurological pain ${ }^{12,33}$. Furthermore, psychosocial dysfunction (that is, depression, anxiety or catastrophizing) was more commonly identified and was associated with more severe UCPPS symptoms and poorer outcomes in patients with comorbid nonurological symptoms than in those without such comorbidities ${ }^{12}$. Thus, greater emphasis should be placed on the early identification and treatment of nonurological symptoms and psychosocial dysfunction as separate and exacerbating clinical factors in UCPPS.

\section{Pain and centrally acting therapies}

MAPP Network studies of structural and functional brain changes in UCPPS have revealed similarities to other chronic pain syndromes and confirmed that the pain experienced by some patients is more complex than simply neuropathy localized to the pelvis ${ }^{51-58}$. Importantly, these observations might explain the favourable responses that some patients with UCPPS have to centrally acting therapies (amitriptyline, pregabalin and sacral nerve stimulation) ${ }^{121,123}$, which is under further investigation in ongoing MAPP II studies, and might provide potential new targets for future medical or even surgical interventions. In MAPP I studies, QST revealed global pain processing abnormalities in participants with UCPPS, particularly in those with concomitant pain beyond the pelvis (S.E.H., H.H.L., S.S., B.D.N. and J.R.L., unpublished observations). The potential utility of neuroimaging 
approaches in clinical management (as opposed to research studies) will need to be further evaluated, but QST is a relatively simple method to apply and might ultimately have clinical utility for patient phenotyping and stratification to personalized therapies (that is, centrally acting therapies for those with centralized pain or peripherally acting therapies for those with non-centralized pain).

\section{Molecular biomarkers for diagnostic algorithms}

The development of molecular biomarkers of UCPPS, although not yet validated for clinical use, has shown promise; initial MAPP Network studies revealed correlations between molecular biomarkers and distinct clinical features, including a relationship between TLR responses and urological pain profiles ${ }^{87,90,92}$. The identification of patient subgroups that overexpress or underexpress specific biomarkers (or a panel of biomarkers) might improve our understanding of the underlying pathophysiological mechanisms, identify new molecular targets, predict response to therapies and add predictive value to UCPPS diagnostic algorithms in the future.

\section{Antibiotic stewardship}

In our clinical experience, the majority of patients with UCPPS have had multiple unsuccessful empirical courses of antibiotics throughout their clinical history without improvement in symptoms. Thus, the routine use of antibiotics in the absence of an identified infection is discouraged ${ }^{124}$. New molecular techniques (for example, cultureindependent NGS or ribosomal RNA (rRNA) gene sequencing and expanded quantitative urine culture) used in MAPP Network studies have the potential to uncover previously unidentified microorganisms involved in UCPPS that would be otherwise missed using standard culture methods. The identification of novel microbial pathobionts, as well as changes in microbial diversity between urine samples from patients with UCPPS and healthy individuals or between patients with distinct symptom profiles, holds great promise in the characterization of novel host-microbial relationships in UCPPS. These insights might promote new approaches to antibiotic treatment or prophylaxis in some patient subgroups.

\section{Conclusions}

The coordinated multisite efforts of the MAPP Research Network have resulted in the extensive characterization of a number of symptom, as well as biological, domains (FIG. 2) in a sizeable cohort of patients with UCPPS and control individuals. This large longitudinal database has identified subsets of patients with UCPPS who have specific phenotypic profiles, suggesting the existence of different mechanisms underlying the pathogenesis of UCPPS symptoms. This heterogeneity might explain the failures of previous clinical trials. MAPP Network studies also indicate that patients with UCPPS who have nonurological COPCs or widespread pain have a more systemic condition with a different natural history and are more likely to exhibit symptom progression and decreased quality of life than those with isolated UCPPS symptoms. No consistent sex differences in the aforementioned relationships were reported, suggesting that IC/BPS (in women and men) and CP/CPPS (in men) are similar conditions. In addition, individuals with UCPPS have a lower overall pain threshold and different brain patterns on fMRI compared with healthy control individuals, 
suggesting that differences in CNS pain processing have a fundamental role in this disorder. These translational studies and findings will rationally inform the next generation of clinical trials for UCPPS. Finally, by revealing these new insights into UCPPS, the MAPP Network has demonstrated the feasibility and benefit of a research approach founded on highly collaborative and integrated studies by investigators with diverse and complementary expertise. Such an approach could serve as a model for the study of other syndromic disorders that have proved difficult to characterize using traditional research strategies.

\section{Supplementary Material}

Refer to Web version on PubMed Central for supplementary material.

\section{Acknowledgements}

Funding for the Multidisciplinary Approach to the Study of Chronic Pelvic Pain (MAPP) Research Network was obtained under a cooperative agreement from the National Institute of Diabetes and Digestive and Kidney Diseases (NIDDK) of the NIH (grants DK82370, DK82342, DK82315, DK82344, DK82325, DK82345, DK82333, DK82316, DK103227, DK103260 and DK103271) with additional funding from the NIH Office of Research on Women's Heath (ORWH). The authors thank the Interstitial Cystitis Association (ICA and Prostatitis Foundation (PF) for their continued support and to the MAPP Research Network advisers serving on our External Experts Panel (EEP), especially W. Bushman. Finally, the authors express their thanks to all the MAPP Research Network investigators and especially to the study participants for their dedication to this effort.

\section{Glossary}

\section{Chronic overlapping pain conditions}

(COPCs). Chronic pain conditions that are known to commonly co-occur in the same patients (for example, urologic chronic pelvic pain syndrome, fibromyalgia, irritable bowel syndrome, chronic fatigue syndrome, vulvodynia, migraine headaches and temporomandibular joint disorders)

\section{Centralized pain phenotype}

A phenotype in patients with chronic pain in which pain has modified the way the central nervous system works such that they have increased sensitivity to stimuli that should hurt but also to normal stimuli (for example, touch, pressure and sounds)

\section{Hunner's lesions}

Painful bladder lesions that are observed in $<10 \%$ of patients with interstitial cystitis/bladder pain syndrome; treatment of these lesions (cautery or steroid injection) often results in symptom relief of variable duration

\section{Ecological momentary assessment}

A research approach that involves assessment of an individual's experiences, behaviours and moods in real time and in their natural environment

\section{T1-weighted imaging}

A type of human MRI that highlights the structure of grey matter in the brain (the location of neuronal cell bodies)

\section{Diffusion tensor imaging}


(DTI). A type of human brain MRI that highlights the structure of white matter (the location of axonal tracts)

\section{Resting-state fMRI}

(rs-fMRI). A type of human brain MRI that highlights activity within and interactions among grey matter regions during the awake resting state

\section{Mechanical allodynia}

A painful sensation caused by innocuous stimuli such as light touch pain originating from a visceral organ (bladder) is 'referred' ('referred mechanical allodynia') to a corresponding dermatome on the skin that shares spinal innervation with the specific visceral organ (suprapubic region). Also known as tactile allodynia

\section{Pathobionts}

Microorganisms that usually do not cause disease but can do so under certain circumstances (for example, via stimulation of the host immune system)

\section{References}

1. Clemens JQ, Markossian TW, Meenan RT, O’Keeffe Rosetti MC \& Calhoun EA Overlap of voiding symptoms, storage symptoms and pain in men and women. J. Urol 178, 1354-1358 (2007). [PubMed: 17706719]

2. Bogart LM, Berry SH \& Clemens JQ Symptoms of interstitial cystitis, painful bladder syndrome and similar diseases in women: a systematic review. J. Urol 177, 450-456 (2007). [PubMed: 17222607]

3. Schaeffer AJ Clinical practice. Chronic prostatitis and the chronic pelvic pain syndrome. N. Engl. J. Med 355, 1690-1698 (2006). [PubMed: 17050893]

4. Held PJ, Hanno PM, Wein a. J., Pauly MV \& Cahn MA in Interstitial Cystitis (eds Hanno PM, Staskin DR, Krane RJ \& Wein AJ ) 29-48 (Springer-Verlag, 1990).

5. Berry SH et al. Prevalence of symptoms of bladder pain syndrome/interstitial cystitis among adult females in the United States. J. Urol 186, 540-544 (2011). [PubMed: 21683389]

6. Suskind AM et al. The prevalence and overlap of interstitial cystitis/bladder pain syndrome and chronic prostatitis/chronic pelvic pain syndrome in men: results of the RAND Interstitial Cystitis Epidemiology male study. J. Urol 189, 141-145 (2013). [PubMed: 23164386]

7. Daniels NA, Link CL, Barry MJ \& McKinlay J B Association between past urinary tract infections and current symptoms suggestive of chronic prostatitis/chronic pelvic pain syndrome. J. Natl Med. Assoc 99, 509-516 (2007). [PubMed: 17534008]

8. Clemens JQ et al. The MAPP Research Network: a novel study of urologic chronic pelvic pain syndromes. BMC Urol. 14, 57 (2014). [PubMed: 25085007]

9. Landis JR et al. The MAPP Research Network: design, patient characterization and operations. BMC Urol. 14, 58 (2014). [PubMed: 25085119]

10. US National Library of Medicine ClinicalTrials.gov https://ClinicalTrials.gov/ct2/show/ NCT01098279 (2014).

11. Lai HH et al. Characterization of whole body pain in urological chronic pelvic pain syndrome at baseline: a MAPP Research Network study. J. Urol 198, 622-631 (2017). [PubMed: 28373134]

12. Krieger JN et al. Relationship between chronic nonurological associated somatic syndromes and symptom severity in urobgical chronic pelvic pain syndromes: baseline evaluation of the MAPP study. J. Urol 193, 1254-1262 (2015). [PubMed: 25444992]

13. Maurer AJ, Lissounov A, Knezevic I, Candido KD \& Knezevic NN Pain and sex hormones: a review of current understanding. Pain Manag. 6, 285-296 (2016). [PubMed: 26983893] 
14. Berry SH et al. Development, validation and testing of an epidemiological case definition of interstitial cystitis/painful bladder syndrome. J. Urol 183, 1848-1852 (2010). [PubMed: 20303099]

15. Lai HH et al. painful bladder filling and painful urgency are distinct characteristics in men and women with urological chronic pelvic pain syndromes: a MAPP Research Network study. J. Urol 194, 1634-1641 (2015). [PubMed: 26192257]

16. Clemens JQ et al. Validation of a modified National Institutes of Health chronic prostatitis symptom index to assess genitourinary pain in both men and women. Urology 74, 983-987 (2009). [PubMed: 19800663]

17. Griffith $\mathrm{W}$ et al. pain and urinary symptoms should not be combined into a single score: psychometric findings from the MAPP Research Network. J. Urol 195, 949-954 (2016). [PubMed: 26585679]

18. O'Leary MP et al. The interstitial cystitis symptom index and problem index. Urology 49, 58-63 (1997). [PubMed: 9146003]

19. Rothrock NE, Lutgendorf SK, Kreder KJ, Ratliff TL \& Zimmerman B Daily stress and symptom exacerbation in interstitial cystitis patients. Urology 57, 122 (2001).

20. Sutcliffe $S$ et al. Urological chronic pelvic pain syndrome flares and their impact: qualitative analysis in the MAPP network. Int. Urogynecol. J 26, 1047-1060 (2015). [PubMed: 25792349]

21. Sutcliffe, et al. Changes in symptoms during urologic chronic pelvic pain syndrome symptom flares: findings from one site of the MAPP Research Network. Neurourol. Urodyn 34, 188-195 (2015). [PubMed: 24273163]

22. Sutcliffe $S$ et al. Urological chronic pelvic pain syndrome symptom flares: characterisation of the full range of fares at two sites in the Multidisciplinary Approach to the Study of Chronic Pelvic Pain (MAPP) Research Network. BJU Int. 114, 916-925 (2014). [PubMed: 24730356]

23. Webster DC \& Brennan T Self-care strategies used for acute attack of interstitial cystitis. Urol. Nurs 15,86-93 (1995). [PubMed: 7481892]

24. Alexander RB \& Trissel D Chronic prostatitis: results of an Internet survey. Urology 48, 568-574 (1996). [PubMed: 8886062]

25. Herati AS et al. Effects of foods and beverages on the symptoms of chronic prostatitis/chronic pelvic pain syndrome. Urology 82 1376-1380 (2013). [PubMed: 23978369]

26. Shorter B, Lesser M, Moldwin RM \& Kushner L Effect of comestibles on symptoms of interstitial cystitis. J. Urol 178, 145-152 (2007). [PubMed: 17499305]

27. Maclure M \& Mittleman MA Should we use a case-crossover design? Annu. Rev. Pubic Heath 21, 193-221 (2018).

28. Sutcliffe, et al. A case-crossover study of urological chronic pelvic pain syndrome fare triggers in the MAPP Research Network. J. Urol 1M, 1245-1251 (2018).

29. Tang YR, Yang WW, Wang YL \& Lin L Sex differences in the symptoms and psychological factors that influence quality of life in patients with irritable bowel syndrome. Eur J. Gastroenterol. Hepatol. 24, 702-707 (2012). [PubMed: 22382707]

30. Poeschla B, Strachan E, Dansie, Buchwald DS \& Afari N Chronic fatigue and personalty: a twin study of causal pathways and shared liabilities. Ann. Behav. Med 45, 289-298 (2013). [PubMed: 23361410]

31. Aaseth $\mathrm{K}$ et al. Personalty traits and psychological distress in persons with chronic tension-type headache. The Akershus study of chronic headache. Acta 124, 375-382 (2011).

32. Naliboff BD et al. Widespread psychosocial difficulties in men and women with urologic chronic pelvic pain syndromes: case-control findings from the multid is cip linary approach to the study of chronic pelvic pain research network. Urology 85, 1319-1327 (2015). [PubMed: 26099876]

33. Naliboff BD et al. Clinical and psychosocial predictors of urological chronic pelvic pain symptom change in 1 year: a prospective study from the MAPP Research Network. J. Urol 198, 848857 (2017).

34. Mordasini L et al. Chronic pelvic pain syndrome in men is associated with reduction of relative gray matter volume in the anterior cingulate cortex compared to healthy controls. J. Urol 188, 2233-2237 (2012). [PubMed: 23083652]

35. Farmer MA et al. Brain functional and anatomical changes in chronic prostatitis/chronic pelvic pain syndrome. J. Urol 186, 117-124 (2011). [PubMed: 21571326] 
36. Apkarian AV, Baliki MN \& Geha PY Towards a theory of chronic pain. Prog. Neurobiol 87, 81-97 (2009). [PubMed: 18952143]

37. Moisset X \& Bouhassira D Brain imaging of neuropathic pain. Neuroimage 37 (Suppl. 1), S80S88 (2007). [PubMed: 17512757]

38. Tracey I. \& Mantyh PW The cerebral signature for pain perception and its modulation. Neuron 55, 377-391 (2007). [PubMed: 17678852]

39. Harris RE \& Clauw DJ How do we know that the pain in fibromyalgia is "real"? Curr. Pain Headache Rep. 10, 403-407 (2006). [PubMed: 17087863]

40. Allsop SA et al. The DABBEC phenotyping system: towards a mechanistic understanding of CP/ CPPS. Nat. Rev. Urol 8, 107-113 (2011). [PubMed: 21243018]

41. Alger JR et al. Multisite, multimodal neuroimaging of chronic urological pelvic pain: methodology of the MAPP Research Network. Neuroimage Clin. 12, 65-77 (2016). [PubMed: 27408791]

42. Martucci KT et al. The posterior medial cortex in urologic chronic pelvic pain syndrome: detachment from default mode network-a resting-state study from the MAPP Research Network. Pain 156, 1755-1764 (2015). [PubMed: 26010458]

43. Kairys AE et al. Increased brain gray matter in the primary somatosensory cortex is associated with increased pain and mood disturbance in patients with interstitial cystitis/painful bladder syndrome. J. Urol 193, 131-137 (2015). [PubMed: 25132239]

44. Farmer MA et al. Brain white matter abnormalities in female interstitial cystitis/bladder pain syndrome: a MAPP network neuroimaging study. J. Urol 194, 118-126 (2015). [PubMed: 25711200]

45. Kilpatrick LA et al. Alterations in resting state oscillatio ns and connectivity in sensory and motor networks in women with interstitial cystitis/painful bladder syndrome. J. Urol 192, 947-955 (2014). [PubMed: 24681331]

46. Bagarinao E et al. Preliminary structural MRI based brain classification of chronic pelvic pain: a MAPP network study. Pain 155, 2502-2509 (2014). [PubMed: 25242566]

47. Kutch JJ et al. Altered resting state neuromotor connectivity in men with chronic prostatitis/chronic pelvic pain syndrome: a MAPP: research network neuroimaging study. Neuroimage Clin. 8, 493502 (2015). [PubMed: 26106574]

48. Huang $\mathrm{L}$ et al. Brain white matter changes associated with urological chronic pelvic pain syndrome: multisite neuroimaging from a MAPP case-control study. Pain 157, 2782-2791 (2016). [PubMed: 27842046]

49. Woodworth D et al. Unique microstructural changes in the brain associated with urological chronic pelvic pain syndrome (UCPPS) revealed by diffusion tensor MRI, super-resolution track density imaging, and statistical parameter mapping: a MAPP network neuroimaging study. PLOS ONE 10, e0140250 (2015). [PubMed: 26460744]

50. Kutch JJ et al. Resting-state functional connectivity predicts longitudinal pain symptom change in urologic chronic pelvic pain syndrome: a MAPP network study. Pain 158, 1069-1082 (2017). [PubMed: 28328579]

51. Asavasopon $\mathrm{S}$ et al. Cortical activation associated with muscle synergies of the human male pelvic floor. J. Neurosci 34, 13811-13818 (2014). [PubMed: 25297107]

52. Rana M, Yani MS, Asavasopon S, Fisher BE \& Kutch JJ Brain connectivity associated with muscle synergies in humans. J. Neurosci 35, 14708-14716 (2015). [PubMed: 26538643]

53. Griffiths D, Tadic SD, Schaefer W \& Resnick NM Cerebral control of the bladder in normal and urge-incontinent women. Neuroimage 37, 1-7 (2007). [PubMed: 17574871]

54. Tadic SD et al. Brain activity underlying impaired continence control in older women with overactive bladder. Neurourol. Urodyn. 31, 652-658 (2012). [PubMed: 22473921]

55. Fowler CJ, Griffiths D \& de Groat WC The neural control of micturition. Nat. Rev. Neurosci. 9 , 453-466 (2008). [PubMed: 18490916]

56. Mai J \& Paxinos G (eds) The Human Nervous System (Elsevier Academic Press, 2012).

57. Kuhtz-Buschbeck JP et al. Cortical representation of the urge to void: a functional magnetic resonance imaging study. J. Urol 174, 1477-1481 (2005). [PubMed: 16145475] 
58. Turnbull GK, Hamdy S, Aziz Q, Singh KD \& Thompson DG The cortical topography of human anorectal musculature. Gastroenterology 117, 32-39 (1999). [PubMed: 10381907]

59. Harper DE et al. Relat ionships between brain metabolite levels, functional connectivity, and negative mood in urologic chronic pelvic pain syndrome patients compared to controls: a MAPP Research Network study. Neuroimage Clin. 17, 570-578 (2018). [PubMed: 29201643]

60 . Kutch JJ et al. Brain signature and functional impact of centralized pain: a multidisciplinary approach to the study of chronic pelvic pain (MAPP) network study. Pain 158, 1979-1991 (2017). [PubMed: 28692006]

61. Kleinhans NM, Yang CC, Strachan ED, Buchwald DS \& Maravilla KR Alterations in connectivity on functional magnetic resonance imaging with provocation of lower urinary tract symptoms: a MAPP Research Network feasibility study of urological chronic pelvic pain syndromes. J. Urol 195, 639-645 (2016). [PubMed: 26497778]

62. Apkarian AV, Bushnell MC, Treede RD \& Zubieta JK Human brain mechanisms of pain perception and regulation in health and disease. Eur. J. Pain 9, 463-484 (2005). [PubMed: 15979027]

63. Gracely RH, Petzke F, Wolf JM \& Clauw DJ Functional magnetic resonance imaging evidence of augmented pain processing in fibromyalgia. Arthritis Rheum. 46, 1333-1343 (2002). [PubMed: 12115241]

64. Ablin K \& Clauw DJ From fibrositis to functional somatic syndromes to a bell-shaped curve of pain and sensory sensitivity : evolut ion of a clinical construct. Rheum. Dis. Clin. North Am 35, 233-251 (2009). [PubMed: 19647139]

65. Fillingim RB Individual differences in pain responses. Curr. Rheumatol. Rep 7, 342-347 (2005). [PubMed: 16174481]

66. Arendt-Nielsen L \& Yarnitsky D Experimental and clinical applications of quantitative sensory testing applied to skin, muscles and viscera. J. Pain 10, 556-572 (2009).

67. Harris RE et al. Comparison of clinical and evoked pain measures in fibromyalgia. J. Pain 7, 521527 (2006). [PubMed: 16814691]

68. Kosek E, Ekholm J \& Hansson P Sensory dysfunction in fibromyalgia patients with implications for pathogenic mechanisms. Pain 68, 375-383 (1996). [PubMed: 9121827]

69. Wilder-Smit h CH \& Robert-Yap J Abnormal endogenous pain modulation and somatic and visceral hypersensitivity in female patients with irritable bowel syndrome. World J. Gastroenterol 13, 3699-3704 (2007). [PubMed: 17659729]

70. Greenspan JD et al. Pain sensitivity risk factors for chronic TMD: descriptive data and empirically identified domains from the OPPERA case control study. J. Pain 12, T61-T74 (2011). [PubMed: 22074753]

71. Maixner W, Fillingim R, Booker D \& Sigurdsson A Sensitivity of patients with painful temporomandibular disorders to experimentally evoked pain. Pain 63, 341-351 (1995). [PubMed: 8719535]

72. Giesecke $\mathrm{T}$ et al. Evidence of augmented central pain processing in idiopathic chronic low back pain. Arthritis Rheum. 50, 613-623 (2004). [PubMed: 14872506]

73. Clauw DJ et al. The relationship between fibromyalgia and interstitial cystitis. J. Psychiatr. Res 31 , 125-131 (1997). [PubMed: 9201654]

74. Fitzgerald MP, Koch D \& Senka J Visceral and cutaneous sensory testing in patients with painful bladder syndrome. Neurourol. Urodyn 24, 627-632 (2005). [PubMed: 16173039]

75. Lai HH, Gardner V, Ness TJ \& Gereau RW 4th Segmental hyperalgesia to mechanical stimulus in interstitial cystitis/bladder pain syndrome: evidence of central sensitization. J. Urol. 191, 12941299 (2014). [PubMed: 24316091]

76. Ness TJ, Lloyd LK \& Fillingim RB An endogenous pain control system is altered in subjects with interstitial cystitis. J. Urol 191, 364-370 (2014). [PubMed: 23973521]

77. Ness TJ, Powell-Boone T, Cannon R, Lloyd LK \& Fillingim RB Psychophysical evidence of hypersensitivity in subjects with interstitial cystitis. J. Urol 173, 1983-1987 (2005). [PubMed: 15879797]

78. Harte SE et al. Development and validation of a pressure-type automated quantitative sensory testing system for point-of-care pain assessment. Med. Biol. Eng. Comput 51, 633-644 (2013). [PubMed: 23381890] 
79. Geisser ME et al. A psychophysical study of auditory and pressure sensitivity in patients with fibromyalgia and healthy controls. J. Pain 9, 417-422 (2008). [PubMed: 18280211]

80. As-Sanie $\mathrm{S}$ et al. Increased pressure pain sensitivity in women with chronic pelvic pain. Obstet. Gynecol 122, 1047-1055 (2013). [PubMed: 24104772]

81. Harte SE et al. Pharmacologic attenuation of cross-modal sensory augmentation within the chronic pain insula. Pain 157, 1933-1945 (2016). [PubMed: 27101425]

82. Dagher A et al. Identification of novel non-invasive biomarkers of urinary chronic pelvic pain syndrome: findings from the multidisciplinary approach to the study of chronic pelvic pain (MAPP) Research Network. BJU Int. 120, 130-142 (2017). [PubMed: 28263447]

83. Pontari MA, Hanno PM \& Ruggieri MR Comparison of bladder blood flow in patients with and without interstitial cystitis. J. Urol 162, 330-334 (1999). [PubMed: 10411032]

84. Kawasaki Y et al. Distinct roles of matrix metalloproteases in the early- and late-phase development of neuropathic pain. Nat. Med 14, 331-336 (2008). [PubMed: 18264108]

85. Kiguchi $\mathrm{N}$ et al. Vascular endothelial growth factor signaling in injured nerves underlies peripheral sensitization in neuropathic pain. J. Neurochem 129, 169-178 (2014). [PubMed: 24304382]

86. Chakraborty S, Kaur S, Guha S \& Batra SK The mult ifaceted roles of neutrophil gelatinase associated lipocalin (NGAL) in inflammation and cancer. Biochim. B iophys. Acta 1826, 129-169 (2012).

87. Schrepf A et al. Inflammation and inflammatory control in interstitial cystitis/bladder pain syndrome: associations with painful symptoms. Pain 155, 1755-1761 (2014). [PubMed: 24907404]

88. Brown J, Wang H, Hajishengallis GN \& Martin M TLR-signaling networks: an integration of adaptor molecules, kinases, and cross-talk. J. Dent. Res 90, 417-427 (2011). [PubMed: 20940366]

89. Milligan ED \& Watkins LR Pathological and protective roles of glia in chronic pain. Nat. Rev. Neurosci 10, 23-36 (2009). [PubMed: 19096368]

90. Schrepf A et al. Toll-like receptor 4 and comorbid pain in interstitial cystitis/bladder pain syndrome: a multidisciplinary approach to the study of chronic pelvic pain research network study. Brain Behav. Immun 49, 66-74 (2015). [PubMed: 25771510]

91. Eller-Smith OC, Nicol AL \& Christianson JA Potential mechanisms underlying centralized pain and emerging therapeutic interventions. Front. Cell Neurosci 12, 35 (2018). [PubMed: 29487504]

92. Schrepf A et al. Inflammation and symptom change in interstitial cystitis or bladder pain syndrome: a multidisciplinary approach to the study of chronic pelvic pain research network study. Urology 90, 56-61 (2016). [PubMed: 26768711]

93. Lv H, Hung CS, Chaturvedi KS, Hooton TM \& Henderson JP Development of an integrated metabolomic profiling approach for infectious diseases research. Analyst 136, 4752-4763 (2011). [PubMed: 21922104]

94. Parker KS et al. Urinary metabolomics identifies a molecular correlate of interstitial cystitis/ bladder pain syndrome in a multidisciplinary approach to the study of chronic pelvic pain (MAPP) Research Network cohort. EBioMedicine 7, 167-174 (2016). [PubMed: 27322470]

95. McLellan LK \& Hunstad DA Urinary tract infection: pathogenesis and outlook. Trends Mol. Med 22, 946-957 (2016). [PubMed: 27692880]

96. Schaeffer AJ, Matulewicz RS \& Klumpp DJ in Campbell-Walsh Urology (eds Wein AJ, Kavoussi LR, Partin AW \& Peters CA) 237-303 (Elsevier, 2016).

97. Whiteside SA, Razvi H, Dave S, Reid G \& Burton JP The microbiome of the urinary tract-a role beyond infection. Nat. Rev. Urol 12, 81-90 (2015). [PubMed: 25600098]

98. Schiffner H [The importance of documentation in emergency medicine]. Z. Arztl. Fortbild. (Jena) 83, 675-676 (1989). [PubMed: 2789455]

99. Nickel JC, Shoskes DA \& Irvine-Bird K Prevalence and impact of bacteriuria and/or urinary tract infection in interstitial cystitis/painful bladder syndrome. Urology 76, 799-803 (2010). [PubMed: 20573386]

100. Warren JW et al. Pilot study of sequential oral antibiotics for the treatment of interstitial cystitis. J. Urol 163, 1685-1688 (2000). [PubMed: 10799160] 
101. Siddiqui H, Nederbragt AJ, Lagesen K, Jeansson SL \& Jakobsen KS Assessing diversity of the female urine microbiota by high throughput sequencing of $16 \mathrm{~S}$ rDNA amplicons. BMC Microbiol. 11, 244 (2011). [PubMed: 22047020]

102. Wolfe AJ et al. Evidence of uncultivated bacteria in the adult female bladder. J. Clin. Microbiol 50, 1376-1383 (2012). [PubMed: 22278835]

103. Siddiqui H, Lagesen K, Nederbragt AJ, Jeansson SL \& Jakobsen KS Alterations of microbiota in urine from women with interstitial cystitis. BMC Microbiol. 12, 205 (2012). [PubMed: 22974186]

104. Abernethy MG et al. Urinary microbiome and cytokine levels in women with interstitial cystitis. Obstet. Gynecol 129, 500-506 (2017). [PubMed: 28178051]

105. Nickel JC et al. Search for microorganisms in men with urologic chronic pelvic pain syndrome: a culture-independent analysis in the MAPP Research Network. J. Urol 194, 127-135 (2015). [PubMed: 25596358]

106. Nickel JC et al. Assessment of the lower urinary tract microbiota during symptom flare in women with urologic chronic pelvic pain syndrome: a MAPP network study. J. Urol 195, 356-362 (2016). [PubMed: 26410734]

107. Ackerman AL et al. Mp29-10 alterations in the urinary fungal mycobiome in patients with bladder pain and urinary urgency. J. Urol 197, e383-e384 (2017).

108. Lai $\mathrm{H}$ et al. Animal models of urologic chronic pelvic pain syndromes: findings from the multidisciplinary approach to the study of chronic pelvic pain research network. Urology 85 , 1454-1465 (2015). [PubMed: 26099889]

109. Lai HH et al. Activation of spinal extracellular signal-regulated kinases (ERK) $1 / 2$ is associated with the development of visceral hyperalgesia of the bladder. Pain 152, 2117-2124 (2011). [PubMed: 21705143]

110. Xu S et al. Transgenic mice expressing MCP-1 by the urothelium demonstrate bladder hypersensitivity, pelvic pain and voiding dysfunction: a multidisciplinary approach to the study of chronic pelvic pain research network animal model study. PLOS ONE 11, e0163829 (2016). [PubMed: 27684718]

111. Rudick CN et al. Host-pathogen interactions mediating pain of urinary tract infection. J. Infect. Dis 201, 1240-1249 (2010). [PubMed: 20225955]

112. Rosen JM \& Klumpp DJ Mechanisms of pain from urinary tract infection. Int. J. Urol 21 (Suppl. 1), 26-32 (2014). [PubMed: 24807489]

113. Rudick CN et al. O-Antigen modulates infection-induced pain states. PLOS ONE 7, e41273 (2012). [PubMed: 22899994]

114. Stemler KM et al. Protamine sulfate induced bladder injury protects from distention induced bladder pain. J. Urol 189, 343-351 (2013). [PubMed: 23174261]

115. Crock LW et al. Central amygdala metabotropic glutamate receptor 5 in the modulation of visceral pain. J. Neurosci 32, 14217-14226 (2012). [PubMed: 23055491]

116. Crock LW et al. Metabotropic glutamate receptor 5 (mGluR5) regulates bladder nociception. Mol. Pain 8, 20 (2012). [PubMed: 22449017]

117. Rudick CN, Chen MC, Mongiu AK \& Klumpp DJ Organ cross talk modulates pelvic pain. Am. J. Physiol. Regul. Integr. Comp. Physiol 293, R1191-R1198 (2007). [PubMed: 17626130]

118. Kim R, Liu W, Chen X, Kreder KJ \& Luo Y Intravesical dimethyl sulfoxide inhibits acute and chronic bladder inflammatio $\mathrm{n}$ in transgenic experimental autoimmune cystitis models. J. Biomed. Biotechnol 2011, 937061 (2011). [PubMed: 21113298]

119. Williams DA Cognitive - behavioral therapy in central sensitivity syndromes. Curr. Rheumatol. Rev 12, 2-12 (2016). [PubMed: 26717953]

120. Sant GR et al. A pilot clinical trial of oral pentosan polysulfate and oral hydroxyzine in patients with interstitial cystitis. J. Urol 170, 810-815 (2003). [PubMed: 12913705]

121. Foster HE Jr. et al. Effect of amitriptyline on symptoms in treatment naive patients with interstitial cystitis/painful bladder syndrome. J. Urol 183, 1853-1858 (2010). [PubMed: 20303115]

122. Propert KJ et al. A prospective study of interstitial cystitis: results of longitudinal followup of the interstitial cystitis data base cohort. J. Urol 163, 1434-1439 (2000). [PubMed: 10751852] 
123. Pontari MA et al. Pregabalin for the treatment of men with chronic prostatitis/chronic pelvic pain syndrome: a randomized controlled trial. Arch. Intern. Med 170, 1586-1593 (2010). [PubMed: 20876412]

124. Hanno PM et al. AUA guideline for the diagnosis and treatment of interstitial cystitis/bladder pain syndrome. J. Urol 185, 2162-2170 (2011). [PubMed: 21497847] 


\section{Key points}

- In urologic chronic pelvic pain syndrome (UCPPS), urological pain and urinary symptoms co-vary, with only moderate correlation, and should be evaluated separately rather than as part of a composite score.

- $\quad$ Participants with UCPPS who report pain beyond the pelvis have more severe UCPPS symptoms and more symptom flares than those with pelvic pain only.

- $\quad$ Participants with UCPPS reported more psychosocial difficulties than painfree healthy control individuals; poor psychosocial functioning in participants with UCPPS was associated with a low likelihood of symptom improvement over time.

- UCPPS involves disturbances in brain-level sensorimotor systems regulating urine storage; these disturbances are powerful enough to produce differences not only in brain function but also in brain structure.

- $\quad$ Different UCPPS symptom profiles are distinguishable by their biological correlates (for example, immune factors).

- Quantitative sensory testing has revealed markedly higher pressure pain sensitivity in participants with UCPPS than in healthy control individuals; high sensitivity was associated with a low likelihood of UCPPS symptom improvement. 
- Urological symptom measures

- Symptom patterns and risk

- Relationship of COPCs

- Patient-centric flare assessment

- Psychosocial factors

- Brain structure and function

- Sensory testing

- Cellular and molecular factors

- Microbiome profiles

- Translational animal models

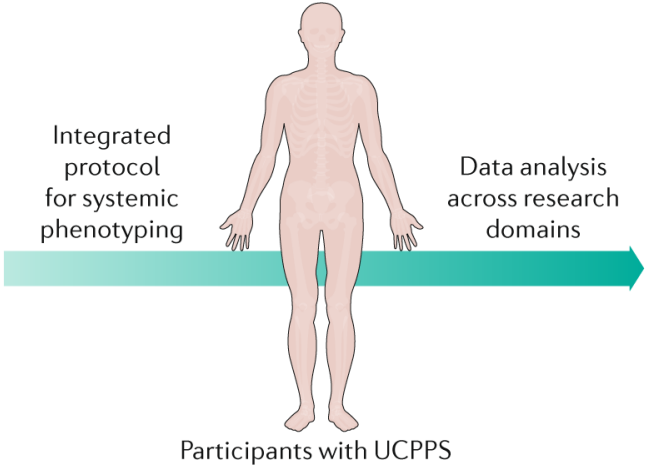

$\checkmark$ Natural history and risk

$\checkmark$ Patient phenotypes

and subgroups

$\checkmark$ Evidence-based disease definitions

$\checkmark$ Improved outcome measures

$\checkmark$ Future clinical trial design

$\checkmark$ Aetiology and mechanism

$\checkmark$ Hypothesis generation

$\checkmark$ Unique research resources

Fig. 1 |. The MAPP Research Network's integrated approach to systemic UCPPS phenotyping. The schematic depicts the Multidisciplinary Approach to the Study of Chronic Pelvic Pain (MAPP) Research Network's novel and highly integrated approach to the study of urologic chronic pelvic pain syndrome (UCPPS). Numerous diverse clinical and biological variables and methods are used to characterize UCPPS in a systemic manner (that is, beyond the traditional sole focus on the urological system) through a common clinical study employed across discovery sites (Trans-MAPP Epidemiology and Phenotyping Study). Analyses that combine data from the study domains (urological, nonurological, psychosocial,

neuroimaging, quantitative sensory testing, molecular phenotyping, microbiome and animal models) are yielding broad new insights into UCPPS. Such insights are expected to directly inform the development of future evidence-based clinical studies (clinical trials) and, ultimately, clinical management, as well as seed future research studies by introducing new investigators with diverse expertise to the study of UCPPS, generating new hypotheses and constructing an unprecedented data and sample resource. COPCs, chronic overlapping pain conditions.

Nat Rev Urol. Author manuscript; available in PMC 2019 October 18. 


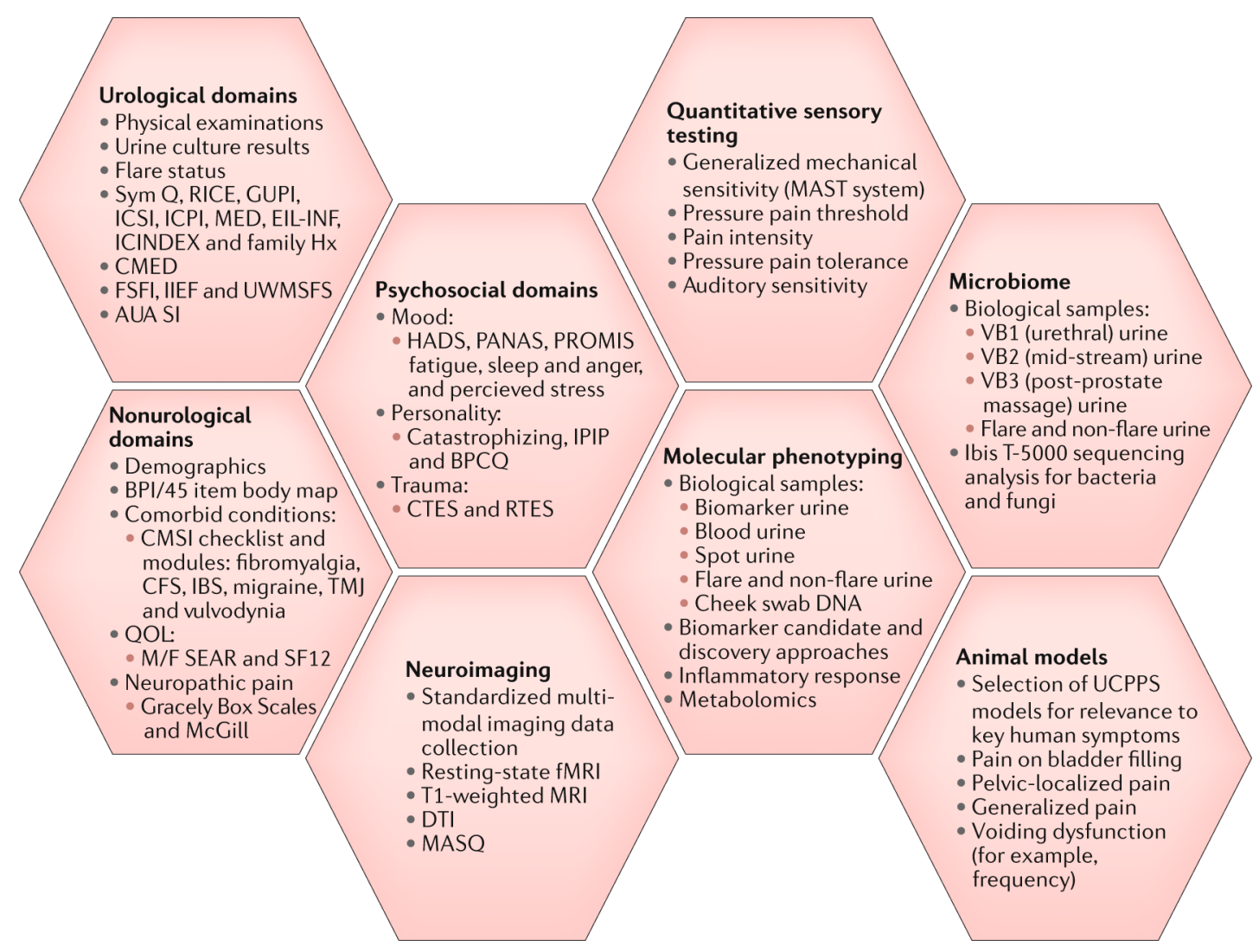

Fig. 2 |. MAPP I data domains and protocol.

In the first phase of studies (MAPP I), the Multidisciplinary Approach to the Study of Chronic Pelvic Pain (MAPP) Research Network collected extensive data describing urologic chronic pelvic pain syndrome (UCPPS) phenotypes. The schematic depicts the various study domains, protocols and associated UCPPS animal models used in the Trans-MAPP Epidemiology and Phenotyping (Trans-MAPP EP) Study ${ }^{9}$. The specific measures collected for each study domain are listed, as well as notations of the main methods and relevant biological samples used, where applicable. Both participants with UCPPS and control individuals were assessed in these broad domains at baseline, and participants with UCPPS were assessed longitudinally during a 12-month follow-up period. AUA SI, American Urologic Association Symptom Index; BPCQ, Beliefs in Pain Control Questionnaire; BPI/45, Brief Pain Inventory-45; CFS, chronic fatigue syndrome; CMED, current medications; CMSI, Complex Medical Symptoms Inventory; CTES, Childhood Traumatic Events Scale; DTI, diffusion tensor imaging; EIL-INF, Early In Life Infection History; Family Hx, family history of UCPPS; fMRI, functional MRI; FSFI, Female Sexual Function Index; GUPI, Genitourinary Pain Index; HADS, Hospital Anxiety and Depression Scale; IBS, irritable bowel syndrome; ICINDEX, Interstitial Cystitis Symptom and Problem Index; ICPI, Interstitial Cystitis Problem Index; ICSI, Interstitial Cystitis Symptom Index; IIEF, International Index of Erectile Function; IPIP, International Personality Item Pool; MASQ, Multiple Ability Self-Report Questionnaire; MAST, multimodal automated sensory testing; MED, medical history; M/F SEAR, Male/Female Self-Esteem and Relationship Questionnaire; PANAS, Positive and Negative Affect Schedule; PROMIS, Patient-Reported 
Outcomes Measurement Information System; QOL, quality of life; RICE, RAND Interstitial Cystitis Epidemiology survey; RTES, Recent Traumatic Events Scale; SF12, 12-item ShortForm Health Survey; Sym Q, MAPP Symptom Questionnaire; TMJ, temporomandibular joint; UWMSFS, University of Washington Male Sexual Function Survey. 
a

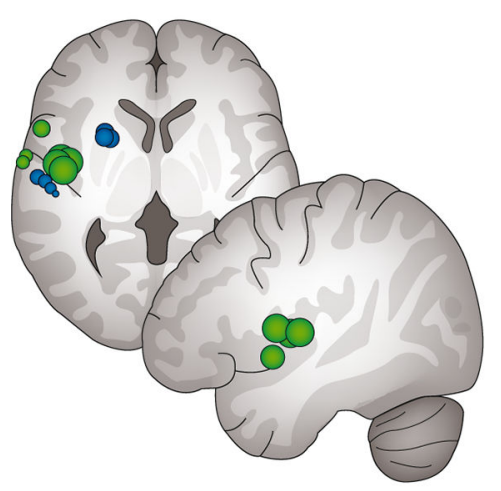

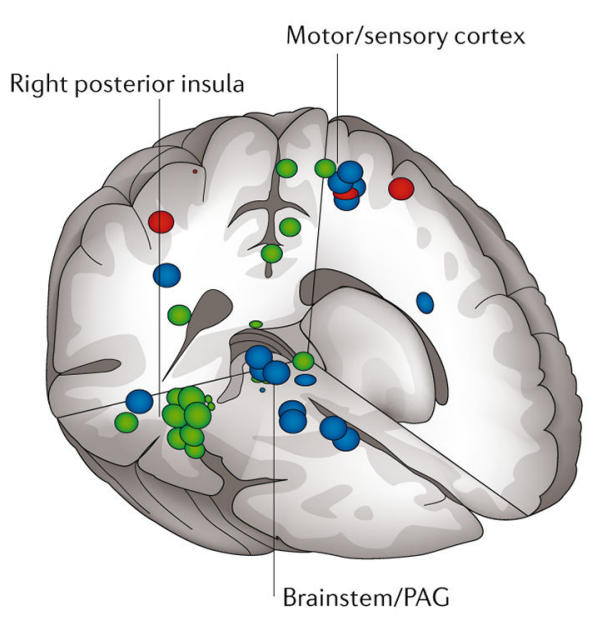

c

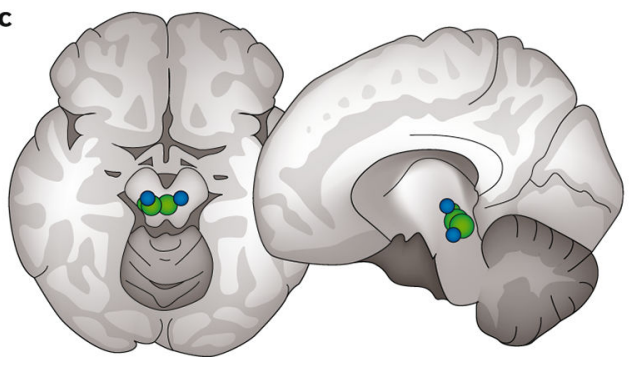

b

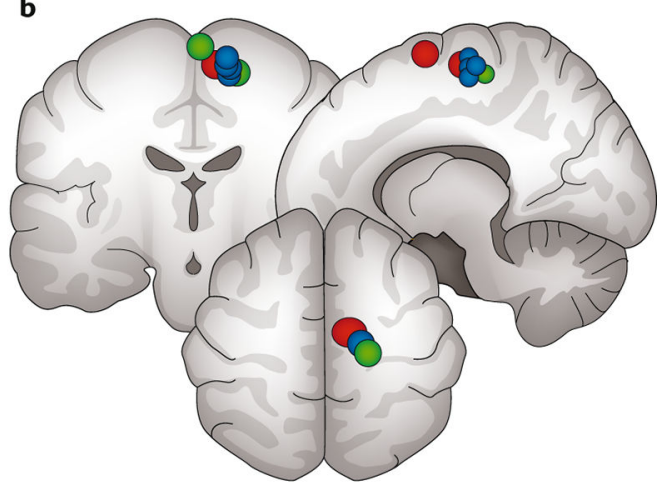

Structural MRI

Diffusion MRI

Functional MRI

Fig. 3 |. MAPP I convergent neuroimaging findings.

Locations in the brain of altered function (green; assessed using resting-state functional MRI), grey matter structure (red; assessed using T1-weighted MRI) and white matter structure (blue; assessed using diffusion tensor MRI) in participants with urologic chronic pelvic pain syndrome (UCPPS) compared with matched healthy control individuals are shown ${ }^{51,52}$. We note particular overlap in the right posterior insula (part a), the medial sensorimotor areas (part b) and the brainstem and periaqueductal grey (PAG) (part c). MAPP, Multidisciplinary Approach to the Study of Chronic Pelvic Pain. 
Table 1

Distribution of key clinical variables among MAPP I participants with UCPPS

\begin{tabular}{|l|l|l|l|}
\hline Sex and total number $(\boldsymbol{n})$ & Pelvic pain only $(\boldsymbol{n}(\boldsymbol{\%}))$ & $\operatorname{Any~COPC}^{\boldsymbol{a}}(\boldsymbol{n}(\boldsymbol{\%}))$ & Bladder-focused symptoms $(\boldsymbol{n}(\boldsymbol{\%}))$ \\
Male $(n=191)$ & $57(29.8 \%)$ & $87(45.6 \%)$ & $144(75.4 \%)$ \\
Female $(n=233)$ & $51(21.9 \%)$ & $137(58.8 \%)$ & $206(88.4 \%)$ \\
Male and female (all patients; $n=424)$ & $108(25.5 \%)$ & $245(57.8 \%)$ & $350(82.6 \%)$ \\
\hline
\end{tabular}

MAPP I, first phase of the Multidisciplinary Approach to the Study of Chronic Pelvic Pain (MAPP) Research Network; UCPPS, urologic chronic pelvic pain syndrome.

${ }^{a}$ Any chronic overlapping pain condition (COPC) includes chronic fatigue syndrome (CFS), fibromyalgia, irritable bowel syndrome (IBS), temporomandibular joint (TMJ) disorders and migraine and is based on Complex Medical Symptoms Inventory (CMSI) diagnostic criteria. 